\title{
Derivation and validation of the clinical prediction model for COVID-19
}

\author{
Fabrizio Foieni ${ }^{1}$. Girolamo Sala ${ }^{1}$. Jason Giuseppe Mognarelli ${ }^{2,4}$. Giulia Suigo ${ }^{3}$. Davide Zampini ${ }^{2}$ - Matteo Pistoia ${ }^{1}$. \\ Mariella Ciola ${ }^{1} \cdot$ Tommaso Ciampani $^{1} \cdot$ Carolina Ultori $^{1} \cdot$ Paolo Ghiringhelli $^{1}$
}

Received: 16 May 2020 / Accepted: 7 July 2020 / Published online: 15 September 2020

(c) The Author(s) 2020

\begin{abstract}
The epidemic phase of Coronavirus disease 2019 (COVID-19) made the Worldwide health system struggle against a severe interstitial pneumonia requiring high-intensity care settings for respiratory failure. A rationalisation of resources and a specific treatment path were necessary. The study suggests a predictive model drawing on clinical data gathered by 119 consecutive patients with laboratory-confirmed COVID-19 admitted in Busto Arsizio hospital. We derived a score that identifies the risk of clinical evolution and in-hospital mortality clustering patients into four groups. The study outcomes have been compared across the derivation and validation samples. The prediction rule is based on eight simple patient characteristics that were independently associated with study outcomes. It is able to stratify COVID-19 patients into four severity classes, with in-hospital mortality rates of $0 \%$ in group 1, 6-12.5\% in group 2,7-20\% in group 3 and $60-86 \%$ in group 4 across the derivation and validation sample. The prediction model derived in this study identifies COVID-19 patients with low risk of in-hospital mortality and ICU admission. The prediction model that the study presents identifies COVID-19 patients with low risk of in-hospital mortality and admission to ICU. Moreover, it establishes an intermediate portion of patients that should be treated accurately in order to avoid an unfavourable clinical evolution. A further validation of the model is important before its implementation as a decision-making tool to guide the initial management of patients.
\end{abstract}

Keywords Covid-19 $\cdot$ Critical illness $\cdot$ Derivation score $\cdot$ Validation score $\cdot$ Score $\cdot$ Predictive-markers $\cdot$ Sars-CoV2

Memorial: This work was made in order to remind people of Roberto Stella (family doctor) and health care workers who gave their lives in the care of COVID-19 patients.

\section{Fabrizio Foieni \\ fabrizio.foieni@asst-valleolona.it \\ Jason Giuseppe Mognarelli giuseppe.mognarelli@unimi.it \\ Girolamo Sala girolamo.sala@asst-valleolona.it}

1 Internal Medicine, Busto Arsizio Hospital, ASST Valle Olona, Busto Hospital, Varese, Lombardy, Italy

2 Vascular Surgery, ASST Valle Olona, Busto Hospital, Varese, Lombardy, Italy

3 Pneumology, ASST Valle Olona, Busto Hospital, Varese, Lombardy, Italy

4 School of Vascular Surgery, Università degli Studi di Milano, Milan, Italy

\section{Introduction}

Coronavirus disease 2019 (COVID-19) is the third coronavirus infection of the past two decades, after severe acute respiratory syndrome (SARS) and Middle East respiratory syndrome (MERS) [1, 2]. As the COVID-19 pandemic spreads worldwide, intensive care unit (ICU) practitioners, hospital administrators, governments, policy makers, and researchers must prepare for a surge in critically ill patients. The number of people with COVID-19 worldwide crossed the one million mark on April 2, 2020; the case fatality rate across 204 countries and territories was 5.2\% [3]. In a large report, $49 \%$ of all 2087 critically ill patients with COVID19 in China died [4, 5]. Small, single-ICU studies found mortality rates of $62 \%$ (in Wuhan, China) and $52 \%$ (in Washington, DC, USA), but these figures had not accounted for many who were still in the ICU. Although $97 \%$ of patients on invasive mechanical ventilation died in a multicenter study conducted early in the Wuhan outbreak, mortality is affected by local practices, and larger studies are awaited 
[6]. Risk stratification in the acute phase of COVID-19 is of paramount importance, because it may help guide decision making in terms of the intensity of the initial treatment during an acute phase and duration of treatment. Prediction of the trajectory of illness from symptom onset is difficult, and prognostic tools and biomarkers are urgently needed. We, therefore, sought to develop a practical clinical prediction rule for patients with covid-19 that identify patients at risk of in-hospital mortality and admission in ICU and that relies only on readily available clinical parameters and ordinary laboratory tests.

\section{Methods}

This study was conducted urgently during the outbreak of COVID-19 in compliance with the declaration of Helsinki, good clinical practice guidelines, and local regulatory requirements (Lombardy, Italy). Written Institutional informed consent about privacy and personal data management was acquired at the presentation to hospital emergency room.

\section{Patient identification and eligibility}

We obtained the medical records and compiled data for 119 consecutive hospitalized patients with laboratory-confirmed COVID-19 from the Busto Arsizio Hospital (Varese, Italy) from 15 March to 30 April 2020. A confirmed case of COVID-19 was defined as a positive result on real-time reverse-transcriptase-polymerase-chain-reaction (RT-PCR) assay of nasal-pharyngeal swab specimens [7]. Alternative respiratory specimen collection in the intubated patient included tracheal aspirates and bronchoscopy alveolar lavage.

\section{Study definitions}

Fever was defined as an axillary temperature of $37.5^{\circ} \mathrm{C}$ or higher. The arterial oxygen saturation in room air (SpO2) was measured on arrival of the patient in Emergency Room with a CE certified Pulse Oximeter Fingertip and at the same time a blood gas analysis was also performed. The C-reactive protein (PCR, $\mathrm{mg} / \mathrm{dL}$ ), Lactate dehydrogenase (LDH, U/L), White blood cell (WBC, $10^{3} / \mathrm{mm}^{3}$ ) count are routine laboratory tests. The $\mathrm{P} / \mathrm{F}$ ratio represents the arterial oxygen pressure $(\mathrm{PaO} 2 \mathrm{in} \mathrm{mmHg})$ to fractional inspired oxygen ( $\mathrm{FiO} 2$ expressed as a fraction, not a percentage). The results used in the rule, where the emergency department (ED) values not the peak values observed during the hospital stay. The ultrasound pattern was carried out in accordance with the use of lung ultrasound for COVID-19 patients proposed by Soldati G et al [19]. We defined "Wet/Interstitial

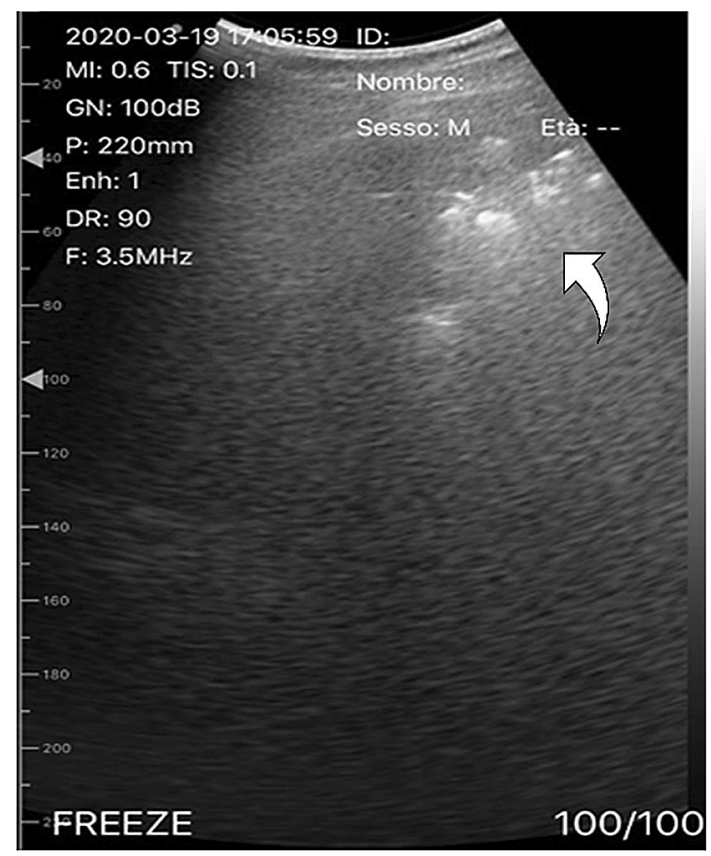

Fig. 1 Pulmonary ultrasound pattern "wet" (the arrow indicates a consolidation with subpleural consolidations)

syndrome" pattern when the operator highlighted B lines, pleura line broken and below the breaking point small to large consolidated areas (score 2 and 3) (Fig. 1); " Dry/Interstitial syndrom" pattern when the pleura line was continuous, regular or indented with visible vertical areas of white below the indent (B lines). B lines reflect local alterations in the acoustical properties of the lung caused by a replacement of air by water, blood, or fibrous tissue [8-10]. Besides, if the "Wet pattern" was localized to one segment of one lung, the whole ultrasound pattern in that patient was considered "Wet".

\section{Laboratory confirmation}

Laboratory confirmation of SARS-CoV-2 was performed at the Virology Laboratory of the University of Milan and at the Virology Laboratory of the San Matteo Hospital in Pavia, Ospedale Niguarda Ca' Granda and Ospedale Maggiore Policlinico in Milan.RT-PCR assays were performed in accordance with the protocol established by the WHO [7].

\section{Baseline predictor variables and outcomes measures}

To derive our prediction rule, we used clinical variables routinely available at presentation that were previously shown to be associated with mortality in patients with Covid-19 or other acute diseases [11]. These variables included demographics, comorbid conditions, physical examination findings, 
and laboratory findings. We extracted the clinical symptoms or signs, and laboratory findings on admission from electronic medical records and all laboratory testing was performed according to the clinical care needs of the patient. Laboratory assessments consisted of a complete blood count, blood chemical analysis, coagulation testing, assessment of liver and renal function, and measures of electrolytes, C-reactive protein and lactate dehydrogenase. The study outcomes used to derive our prediction rule was in-hospital mortality from all cause. We also assessed ICU admission and discharge.

\section{Statistical analysis}

The study cohort consists of 119 consecutive patients with COVID-19 admitted to the same hospital. 79 consecutive patients $(66 \%)$ were selected for the derivation of the model and $40(34 \%)$ for its internal validation. The patients of the derivation cohort were in-patients of the Internal Medicine ward, those including into the validation cohort were mainly in-patients of other COVID-19 departments of the same Hospital. The variables that were previously described as predictors [11] and that were included in the prediction model have been selected by a wider list of variables trough the multiple linear regression. The constant and the $\beta$-coefficients that the regression revealed were used to generate a score for each patient. A clustering of the scores divided patients into four specific groups (group I, group II, group III, group IV). To assess the discriminatory power that the model has to predict outcomes, the study presents a comparison between the ROC curve of each sample, with in-hospital mortality as the positive value. All the analyses have been performed trough Microsoft Excel 2016 and IBM SPSS Software.

\section{Results}

\section{Characteristics of the patients included}

79 patients with COVID-19 were included in derivation sample and 40 patients in the validation sample. Most patients were men (66\%), with a mean age of 68 years (31-91) and 75 (62.5\%) patients suffered from Arterial Hypertension (Table 1); 8 variables were associated with outcomes (Discharge, Admission in ICU, Exitus) by multiple regression analysis. Each weighted variable of the score system is illustrated in Table 2. The prediction rule classified similar proportions of patients in each of the four groups across the derivation and internal validation samples (Table 3).

Table 2 Multivariable predictors of outcomes in the derivation cohort and their respective weights

\begin{tabular}{lclr}
\hline Variables & B-coefficients & 95\%CI & $p$ value \\
\hline Fever for more than 5 days & 0.219 & -0.15 to 0.59 & 0.24 \\
Hypertension & 0.194 & -0.12100 .51 & 0.22 \\
Pattern US "Wet" & 0.731 & $0.42-1.03$ & $<0.001$ \\
P/F ratio & 0.002 & $0.00-0.003$ & 0.02 \\
Lactates (mg/dL) & 0.041 & $0.02-0.06$ & $<0.001$ \\
WBC (G/L) & -0.022 & -0.07 to 0.02 & 0.36 \\
CRP (mg/dL) & 0.019 & $0.00-0.03$ & 0.02 \\
Age & 0.014 & $0.00-0.02$ & 0.02 \\
\hline
\end{tabular}

Table 1 Baseline patient characteristics in the derivation and validation samples

\begin{tabular}{lll}
\hline Patients characteristics & Derivation samples $(n=79)$ & Validation samples $(n=40)$ \\
\hline Age (min-max, mean) & $31-91(66.8)$ & $44-93(71)$ \\
Male sex (\%) & $54(68 \%)$ & $26(65 \%)$ \\
Hypertension (\%) & $56(70 \%)$ & $19(48 \%)$ \\
Temperature $>37.5(\%)$ & $65(82 \%)$ & $31(78 \%)$ \\
Pulmonary pattern "Wet" (\%) & $28(35 \%)$ & $18(45 \%)$ \\
Respiratory rate (min-max, mean, median) & $15-48(26 ; 24)$ & $18-37(24 ; 24)$ \\
Arterial oxygen saturation (min-max, mean, median) & $63-96(88 ; 88)$ & $70-98(90 ; 91)$ \\
Absolute white blood cell count $\left(10^{3} / \mathrm{mm}^{3}\right)$ (min-max, mean, & $1.1-16.1(8.06 ; 7.06)$ & $3.21-21(8,1 ; 6,6)$ \\
$\quad$ median) & & \\
CRP (mg/dL) (min-max, mean, median) & $0.1-41(12.8 ; 11)$ & $0.16-31.5(12 ; 10,5)$ \\
LDH (U/L) (min-max, mean, median) & $87-1602(504 ; 420)$ & $173-1340(418 ; 369)$ \\
BMI (kg/m ${ }^{2}$ (min-max, mean, median) & $18.4-37(26 ; 26)$ & $18-42(27 ; 26)$ \\
P/F Ratio (min-max, mean, median) & $50-460(243 ; 252)$ & $98-453(272 ; 284)$ \\
Lactates $>$ 20 mg/dL (min-max, mean, median) & $2.3-47.7(13.78 ; 11)$ & $5-32(13 ; 11)$ \\
\hline
\end{tabular}

$\mathrm{P} / \mathrm{F}$ ratio, the arterial oxygen pressure divided by the $\mathrm{FIO}_{2}$ (the fraction of inspired oxygen expressed as a decimal). CRP C-reactive protein, $L D H$ lactate dehydrogenase, $B M I$ body mass index 
Table 3 Risk class distribution in the derivation and validation sample

\begin{tabular}{lllll}
\hline Groups (score) & Range formula $^{\mathrm{a}}$ & Range values & $\begin{array}{l}\text { Derivation sample } \\
n(\%)\end{array}$ & $\begin{array}{l}\text { Validation sample } \\
n(\%)\end{array}$ \\
\hline Group 1 & $(\mu-2 \times S d) ;(\mu-S d)$ & $0.3087-0.9391$ & $12(15 \%)$ & $5(12.5 \%)$ \\
Group 2 & $(\mu-S d) ;(\mu)$ & $0.9392-1.5696$ & $33(42 \%)$ & $16(40 \%)$ \\
Group 3 & $(\mu) ;(\mu+S d)$ & $1.5697-2.2000$ & $20(25 \%)$ & $14(35 \%)$ \\
Group 4 & $(\mu+S d) ;(\mu+2 \times S d)$ & $2.2001-2.8304$ & $14(18 \%)$ & $5(12.5 \%)$ \\
\hline
\end{tabular}

${ }^{\mathrm{a}} \mu$ is the scores mean; SD is the scores standard deviation

\section{Determination of item weights and derivation of the Busto Score}

The 8 patient variables independently associated with study outcomes included: fever for more than 5 days, Arterial Hypertension, Pulmonary ultrasound pattern ("wet" or "dry"), P/F index, Lactates, withe blood cells count (WBC), C-reactive protein (CRP) and Age. These clinical and laboratory variables were significantly associated with outcomes in the multiple model, and the R square of the model was 0.567 (Significance $F<0.001$ ). The scoring system shown in Table 2 was used to quantify the magnitude of the association of each of these 8 factors with study outcomes. In the derivation cohort, Busto COVID-19 Score was correlated with "outcomes ", as expected (Pearson value $=0.75$, $p<0,001)$. The procedure to get the score for each patient $\left(\right.$ Score $\left._{\text {patientA }}\right)$ is described by the following formula, where $\vartheta$ is a constant $(-0,998), V_{n}$ is the value of each variable and $\beta_{n}$ is its relative $\beta$-coefficient:

Score $_{p a t i e n t \times A}=\vartheta+V_{1} \times \beta_{1}+V_{2} \times \beta_{2}+\cdots+V_{n} \times \beta_{n}$

To get 4 different groups out of the scores that we obtained we decided to use the standard deviation method.
We considered the mean as the threshold and we created the clusters as described in Table 3. To quickly calculate the score, you can connect to the website www.health-key. it.

\section{Internal validation of the prediction rule}

Once we got the prediction model trough the multivariable regression and the weighted combination of the variables, we run further analysis on the internal validation cohort. The prediction rule classified similar proportions of patients in each of the four groups across the derivation and internal validation samples (Table 4) and in-hospital mortality rate were $22 \%$ and $15 \%$, respectively. Moreover, in each group was not significantly different between the derivation and the validation samples; in the internal validation sample, these rates ranged from $0 \%$ in the group 1 patients to $60 \%$ in the group 4 patients. The rule's discriminatory power for mortality was similar in the derivation and internal validation samples, with an area under the receiver operating characteristic curve (ROC curve) of 0.90 (CI95\% 0.801-0.982) and 0.73 (CI95\% 0.484-0.972), respectively (Fig. 2).
Table 4 Risk class-specific medical outcomes in the derivation and validation samples

\begin{tabular}{llll}
\hline Medical outcomes & Derivation sample $(n=79)$ & Validation sample $(n=40)$ & $p$ value \\
\hline Discharged & $52(66 \%$ of the sample $)$ & $30(75 \%$ of the sample $)$ & 0.85 \\
Group 1 & $12(100 \%)$ & $5(100 \%)$ & \\
Group 2 & $28(84 \%)$ & $11(69 \%)$ & \\
Group 3 & $11(55 \%)$ & $12(85 \%)$ & 0.42 \\
Group 4 & $1(7 \%)$ & $2(40 \%)$ & \\
Admittend in ICU & $9(11 \%$ of the sample $)$ & $4(10 \%$ of the sample $)$ & \\
Group 1 & $0 \%$ & $0 \%$ & 0.12 \\
Group 2 & $3(9 \%)$ & $3(18.75 \%)$ & \\
Group 3 & $5(25 \%)$ & $1(7 \%)$ & \\
Group 4 & $1(7 \%)$ & $6 \%$ & \\
Exitus & $18(22 \%$ of the sample) & $0 \%$ & \\
Group 1 & $0 \%$ & $2(12.5 \%)$ & \\
Group 2 & $2(6 \%)$ & $3(7 \%)$ & \\
Group 3 & $4(20 \%)$ & $3(60 \%)$ & \\
Group 4 & $12(86 \%)$ &
\end{tabular}



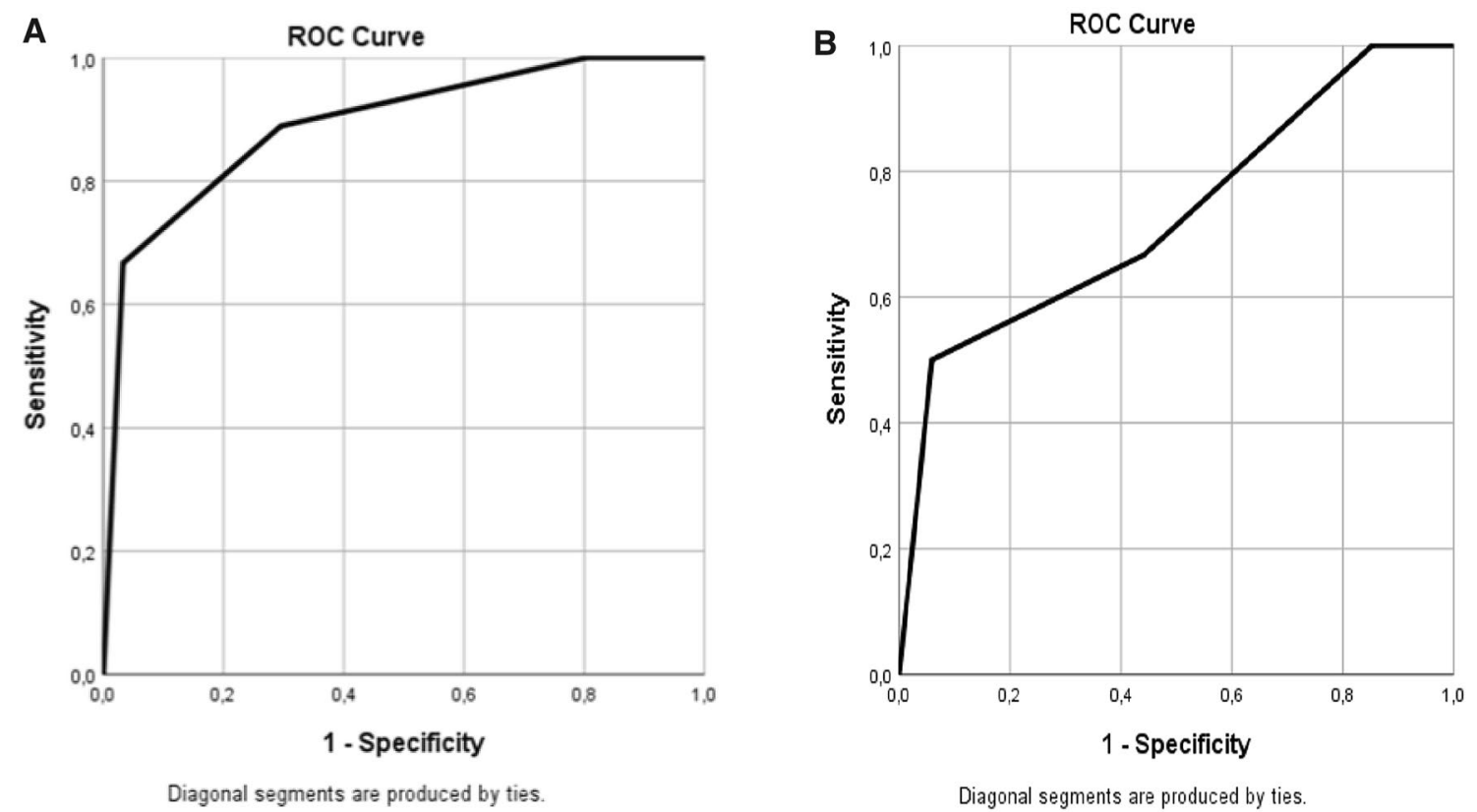

Fig. 2 ROC curves of the derivation (A) and validation sample (B) about outcome "Exitus"

\section{Discussion}

The COVID-19 outbreak put high pressure on Lombardy healthcare services. To prioritize resources for patients with the highest risk mortality, we developed a clinical prediction rule for prognosis of COVID-19 patients and a calculator to allow clinicians to calculate the likelihood (with 95\% CI) that a hospitalized patient could develop critical illness. We identified 8 clinical and laboratory variables that stratify patients into 4 groups with increasing risk of death and other adverse medical outcomes (admission in ICU and mechanical ventilation). Yan L et al. proposed a prediction model to precisely and quickly quantify the risk of death only by laboratory values ( $\mathrm{LDH}, \mathrm{hs}-\mathrm{CRP}$, Lymphocites) [18]. Besides, Liang W et al. developed an online calculator to enter the values of 10 variables including X-ray pattern. Our specific experience allowed us to use, instead of the X-ray pattern, the easier disposable and user-friendly ultrasound tool. The performance of the rule, once validated in a retrospectively identified internal validation sample, was reliable. In the derivation and validation samples, we didn't observe any significant difference between risk groups considering specific mortality, ICU admission and discharge. Our rule accurately identifies patients who are at low risk of fatal medical outcomes: group 1 and group 2 patients, respectively, had $0 \%$ and $6-12 \%$ or less in-hospital mortality. As the current pandemia imposed considerable resource limitations, our rule can provides clinicians with an explicit tool to identifying low-risk patients with COVID-19 who might be potential candidates for outpatient treatment or early hospital discharge. In this subgroup of patients, we observed an in-hospital mortality and an estimated ICU referral of $0 \%$. Anyway, it's mandatory to verify whether selected patients can be safely managed outside the ICU. However, group 4 patients had up to $86 \%$ in-hospital mortality rates and up to $25 \%$ ICU admission and mechanical ventilation rates. The intermediate groups (groups 2 and 3 ) are the most numerous and probably correspond to the overlap subset identified by the Siddiki model [17]. We believe that this is probably the point, where an adequate therapeutic approach can interrupt a process that leads to severe hyperinflammatory syndrome. Our prediction rule has several distinctive strengths: first, it consists of clearly defined and routinely available predictors; second, it concerns a wide spectrum of the disease, ranging from mild symptoms to severe acute respiratory failure with invasive mechanical ventilation. In conclusion, we suggest a practical tool for risk stratification that classifies patients with COVID-19 at increasing risk of death and other adverse outcomes. It can improve outpatient management and early hospital discharge of patients with COVID-19 identified as low risk (group 1 and group 2) with large cost savings without added risk. However, the dataset from a single hospital and the reduced number of cases represent two important limitations of this work. The preliminary results obtained from our experience require further external validation on a larger sample of patients. 


\section{Conclusions}

The prediction model that the study presents identifies COVID-19 patients with low risk of in-hospital mortality and admission to intensive care unit (ICU). Moreover, it establishes an intermediate portion of patients that should be treated accurately to avoid an unfavourable clinical evolution.

Acknowledgements Special thanks to Federico Foieni for supplying data analysis and article construction, and Alessandro Foieni for developing the calculator of Busto COVID 19 Score on the website.

Funding Open access funding provided by Università degli Studi di Milano within the CRUI-CARE Agreement.

\section{Compliance with ethical standards}

\section{Conflict of interest None reported.}

Open Access This article is licensed under a Creative Commons Attribution 4.0 International License, which permits use, sharing, adaptation, distribution and reproduction in any medium or format, as long as you give appropriate credit to the original author(s) and the source, provide a link to the Creative Commons licence, and indicate if changes were made. The images or other third party material in this article are included in the article's Creative Commons licence, unless indicated otherwise in a credit line to the material. If material is not included in the article's Creative Commons licence and your intended use is not permitted by statutory regulation or exceeds the permitted use, you will need to obtain permission directly from the copyright holder. To view a copy of this licence, visit http://creativecommons.org/licenses/by/4.0/.

\section{Bibliography}

1. Morens DM, Daszak P, Taubenberger JK (2020) Escaping Pandora's box-another novel coronavirus. N Engl J Med 382(14):1293-1295. https://doi.org/10.1056/NEJMp2002106

2. Zhu N, Zhang D, Wang W et al (2020) A novel coronavirus from patients with pneumonia in China, 2019. N Engl J Med 382:727-733

3. Worldometer (2020) COVID-19 coronavirus pandemic. https:// www.worldometers.info/coronavirus/ (accessed April 2, 2020)

4. Novel Coronavirus Pneumonia Emergency Response Epidemiology Team. The epidemiological characteristics of an outbreak of 2019 novel coronavirus diseases (COVID-19) in China. Zhonghua Liu Xing Bing Xue Za Zhi 2020; 41: 145-51 (in Chinese).

5. Wu Z, McGoogan JM (2020) Characteristics of and important lessons from the coronavirus disease 2019 (COVID-19) outbreak in China: summary of a report of 72314 cases from the Chinese center for disease control and prevention. JAMA. https://doi. org/10.1001/jama.2020.2648

6. Ruan Q, Yang K, Wang W, Jiang L, Song J (2020) Clinical predictors of mortality due to COVID-19 based on an analysis of data of 150 patients from Wuhan China. Intensive Care Med 46(5):864848. https://doi.org/10.1007/s00134-020-05991-x

7. World Health Organization. Coronavirus disease (COVID-19) technical guidance: laboratory testing for 2019-nCoV in humans. (https://www.who.int/emergencies/diseases/novel-coronaviru s-2019/technical-guidance/laboratory-guidance(accessed March 19, 2020)

8. Huang Y, Wang S, Liu Y et al (2020) A preliminary study on the ultrasonic manifestations of peripulmonary lesions of non-critical novel coronavirus pneumonia (COVID-19). SSR. https://doi. org/10.2139/ssrn.3544750(accessed06/04/2020).2

9. Smith MJ, Hayward SA, Innes SM, Miller ASC (2020) Pointof-care lung ultrasound in patients with COVID-19-a narrative review. Anaesthesia. https://doi.org/10.1111/anae.15082

10. Winkler M, Touw H, van de Ven P, Twisk J, Tuinman PR (2018) Diagnostic accuracy of chest radiograph, and when concomitantly studied lung ultrasound, in critically ill patients with respiratory symptoms: a systematic review and meta-analysis. Crit Care Med 46:e707-e714

11. Henry BM, de Oliveira MHS, Benoit S, Plebani M, Lippi G (2019) Hematologic, biochemical and immune biomarker abnormalities associated with severe illness and mortality in coronavirus disease (COVID-19): a meta-analysis. Clin Chem Lab 58(7):1021-1028. https://doi.org/10.1515/cclm-2020-0369

12. Gattinoni L, Chiumello D, Caironi P et al (2020) COVID-19 pneumonia: different respiratory treatment for different phenotypes? Intensive Care Med. https://doi.org/10.1007/s00134-020-06033-2

13. Marini JJ, Gattinoni L (2020) Management of COVID-19 respiratory distress. JAMA 323(22):2329. https://doi.org/10.1001/ jama.2020.6825

14. Matthay AM, Aldrich JM, Gotts JE (2020) Treatment for severe acute respiratory distress syndrome from COVID-19. Lancet Respir Med. https://doi.org/10.1016/S2213-2600(20)30127-2

15. World Health Organization. (2020) Clinical management of severe acute respiratory infection when novel coronavirus (2019-nCoV) infection is suspected: interim guidance.

16. Sanders JM, Monogue ML, Jodlowsky TZ, Cutrell JB (2020) Pharmacologic treatments for coronavirus disease 2019 (COVID2019). a rewiew. JAMA. https://doi.org/10.1001/jama.2020.6019

17. Siddiqi HK, Mehra MR (2020) COVID-19 illness in native and immunosuppressed states: a clinical-therapeutic staging proposal. J Heart Lung Transplant. https://doi.org/10.1016/j.healu n.2020.03.012

18. Yan L, Zhang H, Goncalves J et al (2020) An interpretable mortality prediction model for COVID-19 patients. Nat Mach Intell 2:283-288. https://doi.org/10.1038/s42256-020-0180-7

19. Soldati $\mathrm{G}$ et al (2020) Lung Ultrasound in COVID Patients. Proposal for international standardization of the use of lung ultrasound for COVID-19 patients; a simple, quantitative, reproducible method. J Ultrasound Med. https://doi.org/10.1002/jum.15285

20. Wynants L, Van Calster B, Bonten MMJ et al (2020) Prediction models for diagnosis and prognosis of covid-19 infection: sistematic review and critical appraisal. BMJ 369:m1328. https:// doi.org/10.1136/bmj.m1328

21. Leidi F, Casella F, Cogliati C (2016) Bedside lung ultrasound in the evaluation of acute decompesated heart failure. Intern Emerg Med. https://doi.org/10.1007/s11739-016-1403-0

22. Trezzi M, Torzillo D, Ceriani E et al (2013) Lung ultrasonography for the assessment of rapid extravascular water variation: evidence from haemodialysis patients. Inten Emerg Med. https:// doi.org/10.1007/s11739-011-0625-4

23. Volpicelli G, Mussa A, Garofalo G et al (2016) Bedside lung ultrasound in the assessment of alveolar-interstitial syndrome. Am J Emerg Med 24(6):689-696. https://doi.org/10.1016/j. ajem.2006.02.013

24. Liang W, Liang H, Ou L et al (2020) Development and validation of a clinical risk score to predict the occurrence of critical illness in hospitalized patients with COVID-19. JAMA Intern Med 180(8):1081. https://doi.org/10.1001/jamainternmed.2020.2033

Publisher's Note Springer Nature remains neutral with regard to jurisdictional claims in published maps and institutional affiliations. 